\title{
Contact Hypersensitivity
}

National Cancer Institute

\section{Source}

National Cancer Institute. Contact Hypersensitivity. NCI Thesaurus. Code C3668.

Contact hypersensitivity is an inflammatory response induced by antigen-reactive T cells that are exposed to antigen on the skin surface (epidermis). Poison ivy is a frequent cause of such reactivity, due to the urushiol from the plant, as are a variety of antigens derived from industrial exposure (e.g. rubber, chromate, nickel). These antigens induce the appearance of Th1-like T cells, which presumably produce IFN to activate macrophages. 